\title{
RETRACTED ARTICLE: Coronary arteries and aortic valve calcifications in COVID-19
}

\author{
Marco Fogante $^{1}\left[\right.$ E $\cdot$ Enrico Cavagna ${ }^{2} \cdot$ Giovanni Rinaldi $^{2}$
}

Received: 2 September 2021 / Accepted: 11 December 2021 / Published online: 23 January 2022

(c) American Society of Emergency Radiology 2021

The Editor in Chief has retracted this article due to multiple simultaneous submissions to other journals [1,2]. Enrico Cavagna and Giovanni Rinaldi agree to this retraction. Marco Fogante does not agree to this retraction.

The online version of this article contains the full text of the retracted article as Supplementary Information.

Supplementary Information The online version contains supplementary material available at https://doi.org/10.1007/s10140-021-02011-3.

\section{References}

1. Fogante M, Cavagna E, Rinaldi G (2021) Coronary arteries and aortic valve calcifications in COVID-19: Not only lungs at chest CT. J Clin Images Med Case Rep. 2(5):1355

2. Fogante M, Cavagna E, Rinaldi G (2022) Correlation between COVID-19 and cardiac calcification. J Med Imaging Radiat Sci

Marco Fogante

marco.fogante89@gmail.com

1 Department of Radiology, Azienda Ospedaliero-

Universitaria “Ospedali Riuniti”, Ancona, Italy

2 Ospedale "Infermi”, Rimini, Italy 